\title{
Limited expression of toll-like receptor 9 on T cells and its functional consequences in patients with nonalco- holic fatty liver disease
}

\author{
Jung Hwan Yu \\ Department of Internal Medicine, Inha University Hospital, Inha University School of Medicine, Incheon, Korea
}

\section{Dear Editor,}

I read the original article titled "Limited expression of TLR9 on T cells and its functional consequences in patients with nonalcoholic fatty liver disease" by Alegre et al.' with great interest. This article studied the role of toll-like receptor (TLR) 9 on T cells in patients with nonalcoholic fatty liver disease (NAFLD) and found positive associations between TLR9 expressions on intrahepatic $\mathrm{CD4}^{+} \mathrm{T}$ cells, necroinflammation, and liver fibrosis. Furthermore, this study revealed associations between TLR9 expression on peripheral $C D 4^{+}$and $C D 8^{+} T$ cells and clinico-pathological alterations of NAFLD, such as body mass index, plasma triglyceride concentration, and aminotransferase activity. In many studies, the pathophysiology of NAFLD has been described as an immune response by either proinflammatory macrophages or T-cell activation via TLRs. ${ }^{2,3}$ I also agree that TLRs play a critical role in regulating immune response and are associated with the pathophysiology of various diseases, including NAFLD. Therefore, it is necessary to evaluate TLR-mediated immune response associated with T-cells in NAFLD patients. However, the pathophysiology of NAFLD is not so simple, and one should always consider the fact that various immune responses and signals work in combination.

TLRs are usually expressed on sentinel cells, such as macro- phages, and are present in humans in the form of subtypes ranging from TLR1 to TLR10. ${ }^{4}$ Although the TLR subtypes play similar roles, each has slightly different functions, and the organs where they are expressed are also slightly different. Therefore, it still remains unclear as to whether TLR plays an important role in NAFLD development. According to previous studies, in addition to TLR9, ${ }^{5}$ other TLRs, such as TLR2, TLR4, and TLR5, ${ }^{6-8}$ have also been found to play a role in the development of NAFLD. However, this study ${ }^{1}$ alone does not reveal which TLRs play a major role in the pathophysiology of NAFLD. In this paper, the question remains as to whether TLR9 is one of the TLRs that play an important role in NAFLD. The authors could have described the role of relative TLR9 in NAFLD more clearly by comparing it with at least one of the other TLRs, such as TLR4.

This article previously stated that TLR9 expression on liver and peripheral T cells is the lowest in patients with simple steatosis, and $T$ cells from patients with simple steatosis induce a limited number of interferon-gamma (IFNY)-producing $C D 8^{+} T$ cells. However, we should consider whether a decrease in TLR9 expression can explain the reduction in IFNy-producing cells. IFNY is known to be an important activator of macrophages and an inducer of class II major histocompatibility complex (MHC) molecule expres-

\section{Abbreviations:}

IFNץ, interferon-gamma; MHC, major histocompatibility complex; NAFLD, nonalcoholic fatty liver disease; NASH, non-alcoholic steatohepatitis; TLR, tolllike receptor

\section{Corresponding author: Jung Hwan Yu}

Department of Internal Medicine, Inha University Hospital, Inha University School of Medicine, 27 Inhang-ro, Jung-gu, Incheon 22332, Korea

Tel: +82-32-890-2548, Fax: +82-32-890-2549

E-mail: junghwan0081@naver.com

https://orcid.org/0000-0001-5672-038X 
sion. In addition, macrophage activity mediated by IFN $\gamma$ has been described as an important mechanism for the development of non-alcoholic steatohepatitis (NASH). ${ }^{9,10}$ In this paper, however, the link between TLR9 and IFNY is somewhat unclear. Although it is clear that TLR is an important factor regulating IFNY, IFNY is itself regulated by several receptors and factors. Without considering various factors that reduce the expression of IFNY, the reduction in IFNy levels may be difficult to explain solely as a result of reduced expression of TLR9 in simple steatosis. In fact, studies have shown that various substances secreted from enteric bacteria of NASH patients affect IFNy production." Therefore, the decrease in TLR9 compression and the decrease in IFNY-producing T cells in this study may have been a phenomenon caused by the complex interactions observed in NAFLD patients. In addition, the possibility of reduced expressions of other TLRs cannot be excluded in patients with simple steatosis.

In conclusion, I also believe that the immune response, including $T$ cells, is important for the pathophysiology of NAFLD, and that the study of TLRs and IFN signaling involved is very important and meaningful. However, this paper did not comment on the effect of other TLRs, and instead focuses on TLR9 only. It also had some limitations in that it did not consider the factors affecting IFNY besides TLR9. Therefore, the limited TLR9 compression and production of IFNy, as concluded in this article, could be a logical leap as to whether it actually plays a role in protecting against simple steatosis. As these avenues have not been explored yet, I do not think that the studies mentioned in this article are wrong; however, I believe further research is needed to provide a better reasoning for the efficient role of TLR9 in NAFLD patients.

\section{Conflicts of Interest}

The author has no conflicts to disclose.

\section{REFERENCES}

1. Alegre NS, Garcia CC, Billordo LA, Ameigeiras B, Poncino D, Benavides J, et al. Limited expression of TLR9 on T cells and its functional consequences in patients with nonalcoholic fatty liver disease. Clin Mol Hepatol 2020;26:216-226.

2. Haas JT, Francque S, Staels B. Pathophysiology and mechanisms of nonalcoholic fatty liver disease. Annu Rev Phys 2016;78:181-205.

3. Petta S, Gastaldelli A, Rebelos E, Bugianesi E, Messa P, Miele L, et al. Pathophysiology of non alcoholic fatty liver disease. Int J Mol Sci 2016;17:2082.

4. Mahla RS, Reddy MC, Prasad DV, Kumar H. Sweeten PAMPs: role of sugar complexed PAMPs in innate immunity and vaccine biology. Front Immunol 2013;4:248.

5. Miura K, Kodama Y, Inokuchi S, Schnabl B, Aoyama T, Ohnishi H, et al. Toll-like receptor 9 promotes steatohepatitis by induction of interleukin-1beta in mice. Gastroenterology 2010;139:323-334.e7.

6. Sharifnia T, Antoun J, Verriere TG, Suarez G, Wattacheril J, Wilson $\mathrm{KT}$, et al. Hepatic TLR4 signaling in obese NAFLD. Am J Physiol Gastrointest Liver Physiol 2015;309:G270-G278.

7. Vijay-Kumar M, Aitken JD, Carvalho FA, Cullender TC, Mwangi S, Srinivasan $\mathrm{S}$, et al. Metabolic syndrome and altered gut microbiota in mice lacking toll-like receptor 5 . Science 2010;328:228-231.

8. Szabo G, Velayudham A, Romics L Jr, Mandrekar P. Modulation of non-alcoholic steatohepatitis by pattern recognition receptors in mice: the role of toll-like receptors 2 and 4 . Alcohol Clin Exp Res 2005;29(11 Suppl):140S-145S.

9. Rau M, Schilling AK, Meertens J, Hering I, Weiss J, Jurowich C, et al. Progression from nonalcoholic fatty liver to nonalcoholic steatohepatitis is marked by a higher frequency of Th17 cells in the liver and an increased Th17/resting regulatory $T$ cell ratio in peripheral blood and in the liver. J Immunol 2016;196:97-105.

10. Yu Y, Liu Y, An W, Song J, Zhang Y, Zhao X. STING-mediated inflammation in Kupffer cells contributes to progression of nonalcoholic steatohepatitis. J Clin Invest 2019;129:546-555.

11. Kolodziejczyk AA, Zheng D, Shibolet O, Elinav E. The role of the microbiome in NAFLD and NASH. EMBO Mol Med 2019;11:e9302. 